\title{
Alteraciones del olfato en enfermedad de Parkinson: validación preliminar de un test diagnóstico en población adulta sana y con síntomas parkinsonianos
}

\author{
Preliminary validation of a smelling test \\ in a sample of Chilean population \\ and its role in the differential diagnosis \\ of Parkinson's disease
}

$\mathrm{S}^{\mathrm{r}}$ r. Editor: El sentido del olfato no es explorado habitualmente en el examen clínico de un paciente, por ser estimado poco relevante para el diagnóstico. Sin embargo, desde hace varias décadas y notoriamente en los últimos 6 años, es creciente el interés científico en la valoración de las alteraciones del olfato como marcadores de enfermedades neurodegenerativas, específicamente las enfermedades de Alzheimer y Parkinson. En ambas y particularmente en la enfermedad de Parkinson, existe una pérdida precoz del olfato, que se presenta incluso décadas antes de aparecer los síntomas motores de la enfermedad ${ }^{1}$. La prevalencia de estas alteraciones olfatorias alcanza de 70 a $100 \%$ de los pacientes parkinsonianos estudiados ${ }^{1,2}$, y a pesar que los pacientes generalmente refieran tener un buen olfato, en $40 \%$ de ellos se ha detectado hiposmia ${ }^{1,2}$. Así, la presencia de hiposmia es tan frecuente como el temblor de reposo, y está en segundo lugar en frecuencia después de la bradikinesia y rigidez, signos clásicos de la enfermedad. El defecto olfatorio es bilateral, aun en presencia de un hemiparkinsonismo, y no es modificado por la terapia dopaminérgica $^{1,2}$. La observación de alteraciones olfatorias

Correspondencia a: Marcelo Miranda. Unidad de Neurología, Clínica Las Condes y Fundación Parkinson y Alzheimer. Lo Fontecilla 441, Santiago. Fax 3650028. E mail: marcelomirandac@gmail.com en un paciente con un cuadro parkinsoniano permite al clínico diferenciar entre un Parkinson primario y aquellos cuadros secundarios a fármacos o de origen vascular, y de otras causas degenerativas, como el Parkinson atípico, trastornos en los que el sentido del olfato está conservado. Incluso se ha propuesto que es posible plantear un patrón de defecto olfatorio específico, en que la pérdida selectiva de ciertos olores (plátano, piña, canela, humo y gasolina) y la conservación de otros (naranja y clavo de olor), serían propios de la enfermedad ${ }^{2}$. Ello no se ha confirmado en otros estudios ${ }^{1}$.

Existen varios tests olfatorios en uso, de los cuales destaca el UPSIT (Test de Investigación del Olfato de la Universidad de Pennsylvania) que evalúa 40 fragancias $^{3}$, principalmente en uso en Estados Unidos y en países de Europa como Inglaterra, y el «Sniffin Stick Test», desarrollado por Hummel en Alemania ${ }^{4}$. Este último consiste en 12 olores comunes que son presentados al sujeto, lo que no demora más de 4 minutos. Se considera anormal reconocer sólo 10 o menos olores ${ }^{4}$.

Sin embargo, ninguno de estos tests ha sido validado en población de origen hispánica, no existe una validación transcultural y étnica. Creemos importante, por sus implicancias diagnósticas, reconsiderar la valoración de este sentido en el estudio de los pacientes neurológicos en que se plantea un Parkinson o un cuadro demencial. El objetivo de esta carta es comunicar nuestra experiencia inicial con el «niffin Stick Test), dar a conocer su reproducibilidad en nuestra población 


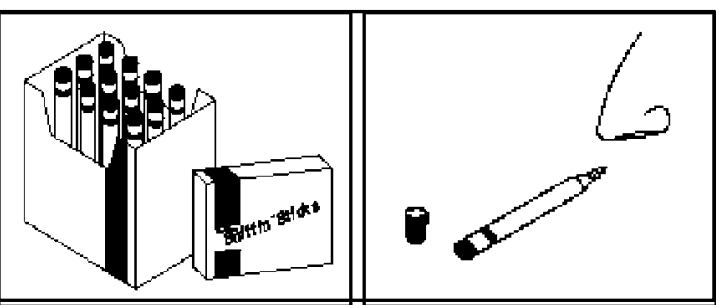

Figura 1. El test de olfato aplicado consistente en 12 〈ápices»con distintos olores a reconocer por el paciente.

adulta sin patología neurológica y comparar sus resultados con un grupo de pacientes con síndrome parkinsoniano idiopático y secundario.

El «niffin Stick Test» consiste en 12 dispositivos con forma de un lápiz ( łápices aromáticos»), que contienen las siguientes esencias de olores disueltos en polipropileno glicol: naranja, limón, piña, cuero, rosa, clavo de olor, menta, pescado, anís, plátano, canela y café (Burghart Medizintechnik, Wedel, Alemania) (Figura 1). Estos lápices son presentados uno a uno por el examinador, a $2 \mathrm{~cm}$ de las fosas nasales del sujeto, y se solicita al paciente que huela e identifique de qué olor se trata, eligiendo entre 4 alternativas posibles presentadas por escrito o leídas por el examinador. Entre cada una de las exposiciones debe existir un intervalo de al menos 30 segundos. Como criterios de exclusión consideramos que el paciente no debía tener patología rinosinusal aguda o crónica, asma bronquial, antecedentes de TEC cerrado con o sin fractura, factores que disminuyen la capacidad olfatoria ${ }^{3,4}$.

Previo consentimiento informado, evaluamos el olfato, específicamente, la capacidad de identifi-

\section{REFERENCIAS}

1. Katzenschlager R, Lees A. Olfaction and Parkinson's disease: its role in differential diagnosis. Curr Opin Neurol 2003; 17: 417-23.

2. HAWKES $\mathrm{CH}$. Olfaction in neurodegenerative diseases. Mov Disord 2003; 18: 364-72.

3. Doty RL, Sharman P, Dann M. Development of the University of Pennsylvania smell identification test. Physiol Behav 1984; 32: 484-502. car olores, de 20 hombres y 20 mujeres, sanos, con edades entre 20 y 85 años y 40 pacientes con Parkinson idiopático, 5 pacientes con parkinsonismo inducido por flunarizina, 4 con la variedad atípica de Parkinson llamada atrofia multisistémica y 1 con parálisis supranuclear progresiva.

Treinta y ocho controles sanos (95\%) tuvieron un reconocimiento de los 12 aromas, sólo 2 reconocieron 10 aromas. En cambio de los pacientes parkinsonianos idiopáticos ninguno alcanzó a reconocer 10 o más olores, es decir 100\% mostró una hiposmia ( $p<0,01)$. Veintisiete pacientes $(67,5 \%)$ reconocieron sólo 6 aromas y 5 pacientes (12,5\%) reconocieron 3 o menos olores. De los pacientes con Parkinson secundario, parálisis supranuclear progresiva y con atrofia multisistémica, todos mostraron una capacidad de discriminar olores comparable a los controles.

Estos hallazgos son concordantes con lo ya comunicado en cuanto a la existencia de un severo defecto olfatorio en los pacientes con enfermedad de Parkinson y que esta evaluación ayuda en el diagnóstico diferencial. El test de lápices olfatorios es una herramienta útil, rápida de aplicar y factible de emplear en nuestro medio. Junto a la ecografía transcraneal, son 2 nuevas herramientas asequibles de que dispone el neurólogo para estudiar un paciente con un cuadro parkinsoniano ${ }^{5}$.

\section{Marcelo Miranda $C^{1}$, Andrea Slachevsky $\mathrm{Ch}^{1,2}$, Carolina Pérez J1a.}

${ }^{1}$ Departamento de Neurología, Clínica Las Condes. Santiago, Chile.

apsicóloga

4. Hummel T, Sekinberg B, Wolf SR. «Sniffin sticks». olfactory performance by the combined testing of odor identification, odor discrimination and olfactory threshold. Chem Senses 1997; 22: 39-52.

5. Miranda M. Ecografía Transcraneal: una nueva herramienta útil en el diagnóstico de la enfermedad de Parkinson [Carta al Editor]. Rev Méd Chile 2006; 134: 525-7. 